\title{
Spatial correlations of elementary relaxation events in glass-forming liquids
}

\author{
Raffaele Pastore $^{1, *}$, Antonio Coniglio ${ }^{1}$, and Massimo Pica Ciamarra ${ }^{2,1}$ \\ ${ }^{1}$ CNR-SPIN, Dipartimento di Scienze Fisiche, Universitá di Napoli Federico II, Italy \\ ${ }^{2}$ Division of Physics and Applied Physics, School of Physical and Mathematical Sciences, \\ Nanyang Technological University, Singapore \\ *Corresponding author: pastore@na.infn.it
}

\begin{abstract}
The dynamical facilitation scenario, by which localized relaxation events promote nearby relaxation events in an avalanching process, has been suggested as the key mechanism connecting the microscopic and the macroscopic dynamics of structural glasses. Here we investigate the statistical features of this process via the numerical simulation of a model structural glass. First we show that the relaxation dynamics of the system occurs through particle jumps that are irreversible, and that cannot be decomposed in smaller irreversible events. Then we show that each jump does actually trigger an avalanche. The characteristic of this avalanche change on cooling, suggesting that the relaxation dynamics crossovers from a noise dominated regime where jumps do not trigger other relaxation events, to a regime dominated by the facilitation process, where a jump trigger more relaxation events.
\end{abstract}




\section{Introduction}

Structural glasses, which are amorphous solids obtained by cooling liquids below their melting temperature avoiding crystallization, provide an array of questions that has been challenging researchers in the last decades $1: 2 ; 3$. These include the nature of the glass transition, the origin of the extraordinarily sensitivity of the relaxation time on temperature, the Boson-peak, the relaxation dynamics. In this respect, here we consider that there is not yet an established connection between the short time single particle motion, and the overall macroscopic dynamics. When observed at the scale of a single particle, the motion of structural glasses is well known to be intermittent. This is commonly rationalized considering each particle to rattle in the cage formed by its neighbors, until it jumps to a different cage ${ }^{4}$. Conversely, when the motion is observed at the macroscale, a spatio-temporal correlated dynam-

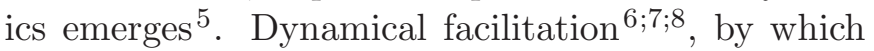
a local relaxation event facilitates the occurrence of relaxation events in its proximity, has been suggested as a key mechanism connecting the microscopic and the macroscopic dynamics. Indeed, kinetically constrained lattice model $\underline{\underline{9}}$, which provide the conceptual framework of the dynamical facilitation scenario, reproduce much of the glassy phenomenology and are at the basis of a purely dynamical interpretation of the glass transition. Different numerical approaches have tried to identify irreversible relaxation events 10;11;12;13;14;15;16;17;18;19:20, and both numerical $21: 22$ and experimental works $\underline{23: 24}$ revealed signatures of a dynamical facilitation scenario.

Here we provide novel insights into the dynamical facilitation mechanisms through the numerical investigation of a model glass former. We show that it is possible to identify single particle jumps that are $\mathrm{el}$ ementary relaxations, being short-lasting irreversible events that cannot be decomposed in a sequence of smaller irreversible events. We then clarify that these jumps lead to spatio-temporal correlations as each jump triggers subsequent jumps in an avalanch- ing process. The statistical features of the avalanches changes on cooling. Around the temperature where the Stokes-Einstein relation first breaks down, the dynamics shows a crossover from a high temperature regime, in which the avalanches do not spread and the dynamics is dominated by thermal noise, to a low temperature regime, where the avalanches percolate. These results suggest to interpret dynamical facilitation as a spreading process $\frac{25}{2}$, and might open the way to the developing of dynamical probabilistic models to describe the relaxation of glass formers.

\section{Methods}

We have performed NVT molecular dynamics simulations $\underline{26}$ of a two-dimensional 50:50 binary mixture of $2 N=10^{3}$ of disks, with a diameter ratio $\sigma_{L} / \sigma_{S}=1.4$, known to inhibit crystallization, at a fixed area fraction $\phi=1$ in a box of side $L$. Particles interact via an soft potential $27, V\left(r_{i j}\right)=$ $\epsilon\left(\left(\sigma_{i j}-r_{i j}\right) / \sigma_{L}\right)^{\alpha} \Theta\left(\sigma_{i j}-r_{i j}\right)$, with $\alpha=2$ (Harmonic). Here $r_{i j}$ is the interparticle separation and $\sigma_{i j}$ the average diameter of the interacting particles. This interaction and its variants (characterized by different values of $\alpha$ ) are largely used to model dense colloidal systems, such as foams $\frac{28}{}$, microgels $\frac{29}{}$ and glasses $30 ; 31$. Units are reduced so that $\sigma_{L}=m=\epsilon=k_{B}=1$, where $m$ is the mass of both particle species and $k_{B}$ the Boltzmann's constant. The two species behave in a qualitatively analogous way, and all data presented here refer to the smallest component.

Cage-jump detection algorithm. We segment the trajectory of each particle in a series of cages interrupted by jumps using the algorithm of Ref. $\stackrel{32}{ }$, following earlier approaches $\frac{11}{}$. Briefly, we consider that, on a timescale $\delta$ of few particle collisions, the fluctuation $S^{2}(t)$ of a caged particle position is of the order of the Debye-Waller factor (DWF) $\left\langle u^{2}\right\rangle$. By comparing $S^{2}(t)$ with $\left\langle u^{2}\right\rangle$ we therefore consider a particle as caged if $S^{2}(t)<\left\langle u^{2}\right\rangle$, and as jumping otherwise. Practically, we compute $S^{2}(t)$ as $\left\langle\left(r(t)-\langle r(t)\rangle_{\delta}\right)^{2}\right\rangle_{\delta}$, where the averages are computed in the time interval 
$[t-\delta: t+\delta]$, with $\delta \simeq 10 t_{b}$, and $t_{b}$ is the ballistic time. At each temperature DWF is defined according to Ref. $\stackrel{33}{,},\left\langle u^{2}\right\rangle=\left\langle r^{2}\left(t_{D W}\right)\right\rangle$, where $t_{D W}$ is the time of minimal diffusivity of the system, i.e. the time at which the derivative of $\log \left\langle r^{2}(t)\right\rangle$ with respect to $\log (t)$ is minimal. At each instant the algorithm allows to identify the jumping particles and the caged ones. We stress that in this approach a jump is a process with a finite duration. Indeed, by monitoring when $S^{2}$ equals $\left\langle u^{2}\right\rangle$, we are able to identify the time at which each jump (or cage) starts and ends. We thus have access to the time, $t_{p}$, a particle persists in its cage before making the first after an arbitrary chosen $t=0$ (persistence time), to the waiting time between subsequent jump of the same particle $t_{w}$ (cage duration), and to the duration $\Delta t_{j}$ and the length $\Delta r_{J}$ of each jump.

\section{Results}

\subsection{Jumps as irreversible elementary pro- cesses}

The idea of describing the relaxation of structural glasses as consisting of a sequence of irreversible processes is not new, and different approaches have been followed to identify these events. For instance, irreversible events have been associated to change of neighbors $\underline{13}: 14 ; 15$, to displacements overcoming a threshold in a fixed time laps 22 , to processes identified through clustering algorithm applied to the particle trajectories $21: 23$, or to more sophisticated approaches $\frac{17}{}$. We notice that since at long time particles move diffusively, all procedures that coarse grains the particle trajectory enough will eventually identify irreversible events. Here we show that the jumps we have identified are irreversible, and we give evidence suggesting that these can be considered as 'elementary' irreversible events, i.e that they are the smallest irreversible single-particle move, at least in the range of parameters we have investigated.

Investigating both the model considered here $\underline{32}$, as well as the $3 \mathrm{~d}$ Kob-Andersen Lennard-Jones (3d

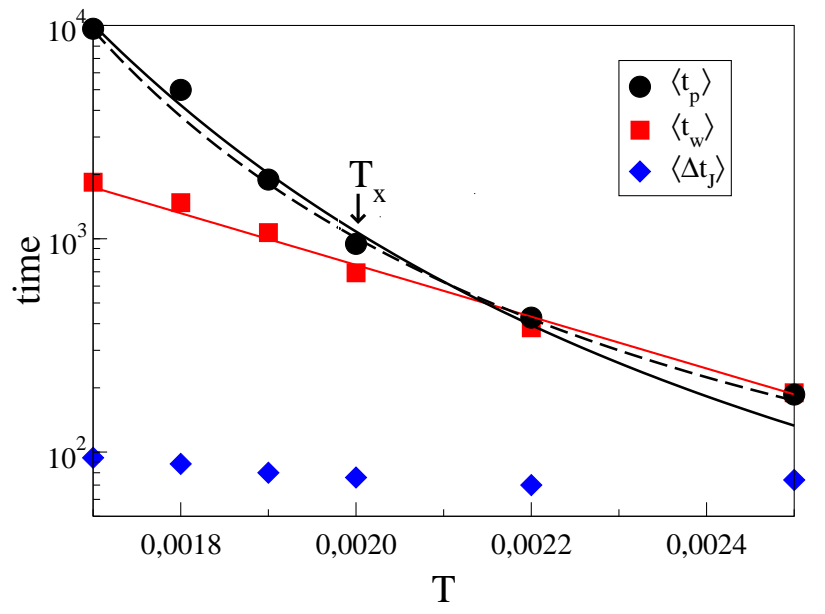

Figure 1: Average persistence time, $\left\langle t_{p}\right\rangle$, cage duration, $\left\langle t_{w}\right\rangle$ and jump duration, $\left\langle\Delta t_{J}\right\rangle$, as a function of the temperature. $\left\langle t_{w}\right\rangle$ grows as an Arrhenius $\left\langle t_{w}\right\rangle \propto \exp (A / T)$ (red full line), whereas $\left\langle t_{p}\right\rangle$ is compatible with several super-Arrhenius laws. The black full line is, for example, a fit $\left\langle t_{p}\right\rangle \propto \exp \left(A / T^{2}\right)$, while the black dashed line is a Vogel-Fulcher law $\left\langle t_{p}\right\rangle \propto \exp \left(B /\left(T-T_{0}\right)\right)$, predicting a divergence at a finite temperature $T_{0} \simeq 0.001$. The arrow indicates the temperature $T_{x}=0.002$ where $\left\langle t_{p}\right\rangle$ and $\left\langle t_{w}\right\rangle$ decouple and the SE relation breaks down. Conversely, $\left\langle\Delta t_{J}\right\rangle$ remains roughly constant on cooling.

KA LJ) binary mixture $\underline{34}$ and experimental colloidal glass $\frac{35}{}$, we have previously shown that the protocol defined in Sec. 2 leads to the identification of irreversible events. Indeed, the mean square displacement of the particles increases linearly with the number of jumps, allowing to describe the dynamics as a continuous time random walk (CTRW) ${ }^{36}$.

Within this approach two fundamental timescales are found, the average persistence time $\left\langle t_{p}\right\rangle$ and the average cage duration $\left\langle t_{w}\right\rangle$. The former corresponds to the relaxation time at the wavelength of the order of the jump length $\left\langle\Delta r_{J}\right\rangle$, while the latter is related to the self diffusion constant, $D \propto\left\langle\Delta r_{J}^{2}\right\rangle /\left\langle t_{w}\right\rangle$. Fig 1 shows that the two timescales are equal at high temperature, but decouple at a temperature $T_{x} \simeq 0.002$, 


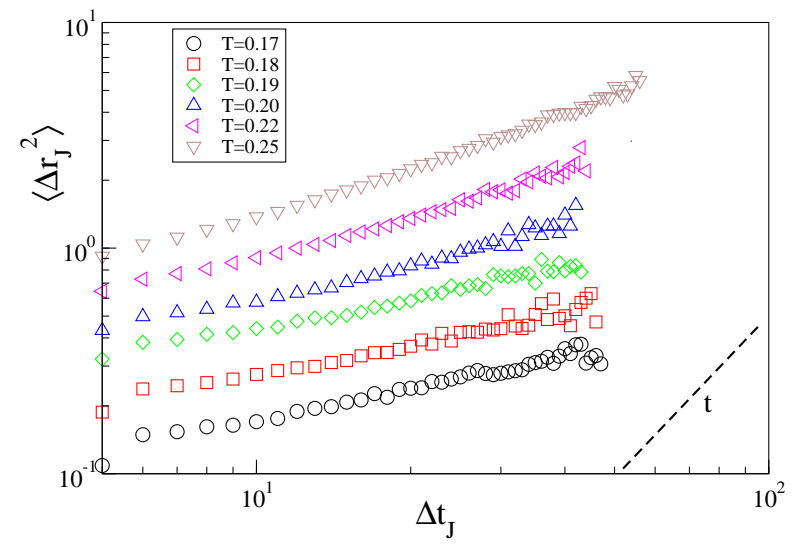

Figure 2: Mean squared jump length $\left\langle\Delta r_{J}^{2}\right\rangle$ as a function of the jump duration $\Delta t_{J}$ at different temperatures.

which marks the onset of the Stokes-Einstein (SE) breakdown at the wavelength of the jump length. We find that $\left\langle t_{w}\right\rangle$ shows an Arrhenius temperature dependence $\left\langle t_{w}\right\rangle \propto \exp (A / T)$, while $\left\langle t_{p}\right\rangle$ increases with a faster super-Arrhenius behaviour (see the caption of Fig(1). It is worth noticing that the decoupling between the average persistence and waiting time, is known to control the breakdown of the SE relation at generic wavelengths, and to induce temporal heterogeneities $37: 34$. These findings suggest that $T_{x}$ may represent a crossover from a localized to a more correlated relaxation process. A similar scenario has been recently reported for models of atomic glass forming liquids, where the SE breaks down and the size of dynamics heterogeneities markedly accelerates below a well defined value of $T_{x} \cdot \underline{\underline{38}}$

We performed two investigations supporting the elementary nature of the jumps we have identified. First, we have considered the change of the average jump duration $\left\langle\Delta t_{J}\right\rangle$ on cooling, as the duration of elementary relaxations is expected not to grow with the relaxation time. Fig. 1 shows that the $\left\langle\Delta t_{J}\right\rangle$ is essentially constant, despite the relaxation time $\left\langle t_{p}\right\rangle$ varying by order of magnitudes. Indeed, at low temperature $\left\langle t_{p}\right\rangle /\left\langle\Delta t_{J}\right\rangle \gg 1$, clarifying why we call them 'jumps'. Then we have considered how parti-

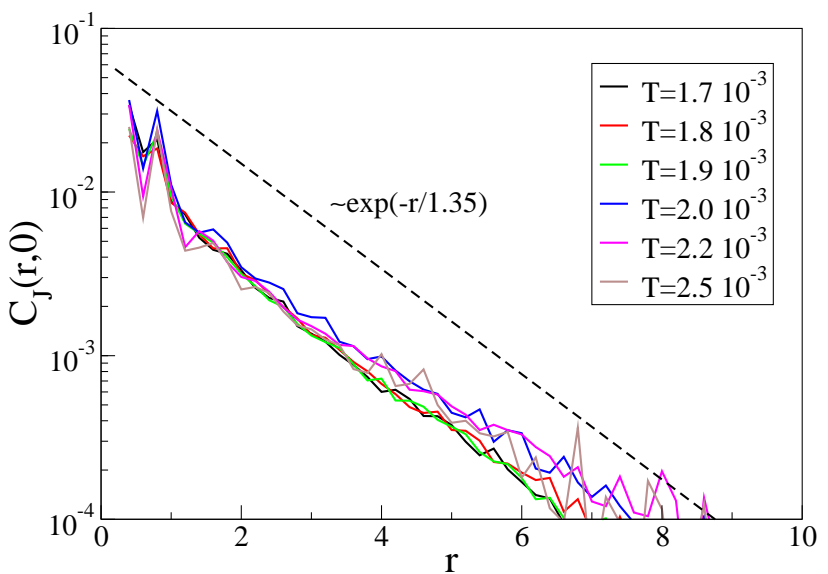

Figure 3: Excess probability to observe contemporary jumps, $C_{J}(r, 0)$, as function of the distance and at different temperature, as indicated. The dashed line is a guide to the eyes $\propto \exp (-1.35 / r)$.

cles move while making a jump. Fig. 2 illustrates that the mean squared jump length grows subdiffusively as a function of the jump duration, with a subdiffusive exponent that decreases on cooling. Conversely, one would expect a diffusive behaviour if jumps were decomposable in a series of irreversible steps.

These results supports the identification of the jumps we have defined with the elementary relaxations leading to the macroscopic relaxation of the particle system.

\subsection{Correlations between jumps}

While each particle behaves as a random walker as it performs subsequent jumps, yet jumps of different particles could be spatially and temporally correlated. We investigate these correlations focusing on the properties of a jump birth scalar field, defined as

$$
b(r, t)=\frac{1}{N} \sum_{i}^{N} b_{i}(t) \delta\left(r-r_{i}(t)\right) .
$$

Here $b_{i}(t)=1$ if particle $i$ starts a jump between $t$ and $t+\delta t$, where $\delta t$ is our temporal resolution, $b_{i}(t)=$ 
0 otherwise. The scalar field $b$ allows to investigate the statistical features of the facilitation process by which a jump triggers subsequent ones. To this end, we indicate with $\langle b(r, t)\rangle_{b(0,0)=1}$ the probability that a jump starts in $(t, r)$ given a jump in $(t=0, r=0)$, and investigate the correlation function

$$
C_{J}(r, t)=\left[\frac{\langle b(r, t)\rangle_{b(0,0)=1}-\langle b\rangle}{g(r, t)}\right] .
$$

Here $g(r, t)$ is a time dependent generalization of the radial distribution function

$$
g(r, t) d r=\frac{1}{2 \pi r \rho(N-1)} \sum_{i \neq j} \delta\left(r-\left|r_{j}(t)-r_{i}(0)\right|\right),
$$

through which we avoid the appearance of spurious oscillations in the correlation function $C_{J}(r, t)$ due to the short range ordering of the system. In Eq2, $\langle b\rangle$ is the spatio-temporal average of the jump birth, and decreases on cooling as $\langle b\rangle=\left(\left\langle t_{w}\right\rangle+\left\langle\Delta t_{J}\right\rangle\right)^{-1}$ (at low temperature $\langle b\rangle \simeq\left\langle t_{w}\right\rangle^{-1}$ as $\left\langle t_{w}\right\rangle<\left\langle\left\langle\Delta t_{J}\right\rangle\right.$ ). Accordingly, the correlation function $C_{J}(r, t)$ is the probability that a jump triggers a subsequent one at a distance $r$ after a time $t$.

We first consider the spatial correlations between contemporary jumps, where two jumps are considered contemporary if occurring within our temporal resolution. Fig. 3 shows that $C_{J}(r, 0)$ decays exponentially, with a temperature independent correlation length $\xi_{J}(0, T) \simeq 1.35$. This result clarifies that jumps aggregate in cluster of roughly $N_{\text {corr }} \simeq$ $\rho \pi \xi_{J}^{2}(0) \simeq 5$ events. A similar scenario has been observed in a different model system, where jumps have been observed to aggregate in clusters of roughly 7.6 particles 21 . Our results also support previous findings suggesting 22 that the elementary excitations of structural glasses have a temperature-independent length not larger than few particle diameters and are consistent with a recently introduced first principle extension of the Mode Coupling Theory $\underline{\underline{39}}$. The investigation of the displacements of the particle jumping in each cluster does not reveal characteristic spatial features. Structured particle motion, such as string-like particle displacements $\underline{40}$ or displacements
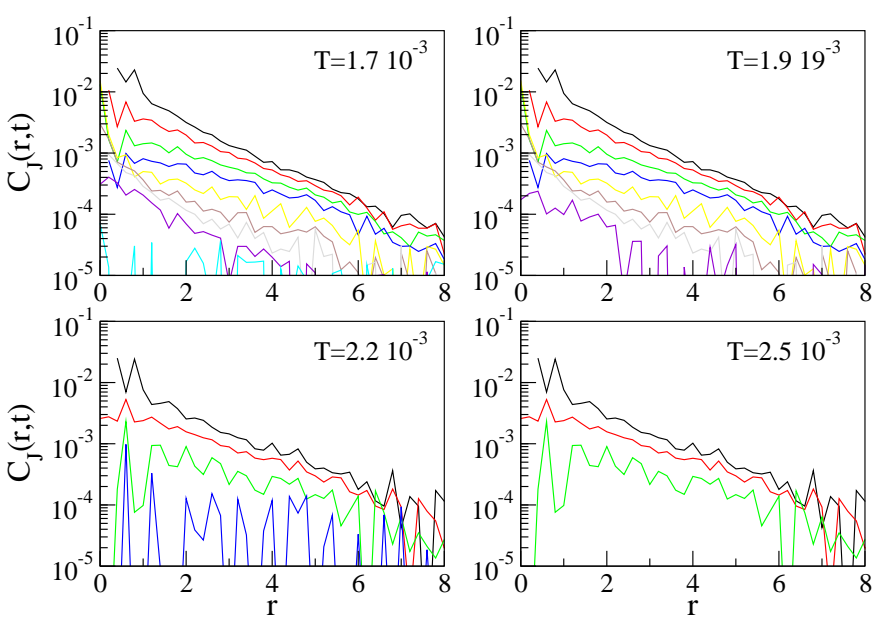

Figure 4: Evolution of the spatial correlation between jumps with time. Each panel refer to a different temperature, as indicated. Within each panel, the different curves correspond to $t=$ $0,10,20,30,100,500,10^{3}$ and $10^{5}$, from top to bottom. At high temperature data corresponding to the largest times are missing as the correlation is too small to be measured.

reminiscent of $\mathrm{T} 1$ events $\underline{41}$ must therefore result from a succession of events rather than a single one.

We now consider the time evolution of the spatial correlation between jumps. Fig. 4 illustrates that at all temperatures and times the decay of the correlation function is compatible with an exponential, $C_{J}(r, t) \propto A(t) \exp \left(-r / \xi_{J}(t)\right)$. The time dependence of the amplitude is illustrated in Fig. 5 . At all temperatures the short time decay of the amplitude is exponential, $A(t, T)=A(0, T) \exp \left(-t / \tau_{A}(T)\right)$, the characteristic decay time slightly increasing on cooling. While no other decay is observed at high temperatures, at low temperatures the exponential decay crossovers towards a much slower power-law decay $A(t) \sim t^{-a}$, with $a \simeq 0.4$. Fig. 6 shows that the correlation length slowly grows in time, approximately as $\xi_{J}(t) \sim t^{b}$, with $b \simeq 0.1$.

The initial fast decrease of the amplitude makes difficult to obtain reliable estimates of its time de- 

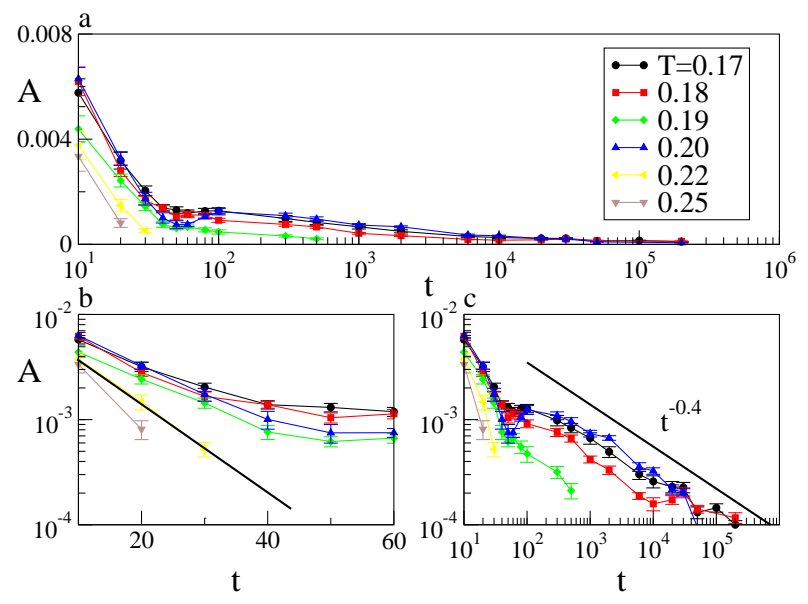

Figure 5: Panel a shows that the amplitude $A(t)$ of the jump correlation function $C_{J}(r, t)$. Panels b and c clarify that a first exponential decay is followed, at low temperature, by a second one, which approximately follows a power law.

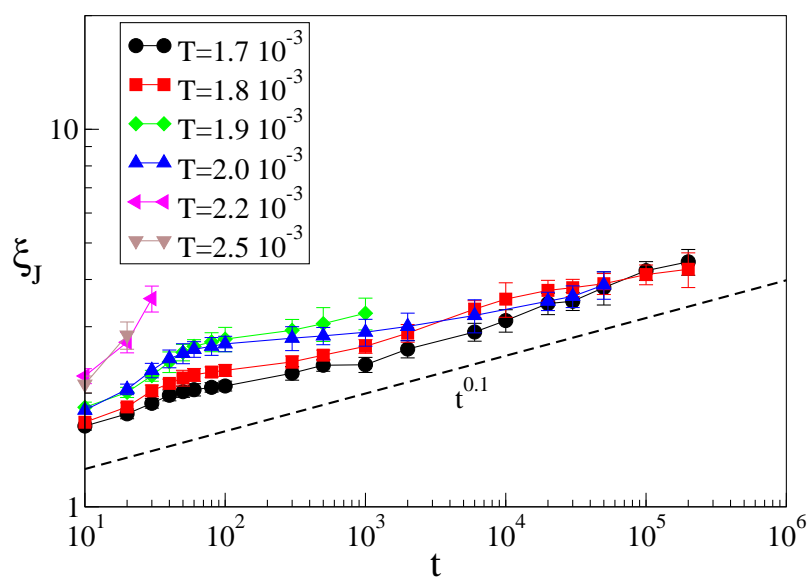

Figure 6: Time dependence of the jump correlation length, at different temperatures. The data suggest that at low temperature the correlation length slowly grows in time, as $\xi_{J}(T) \propto t^{0.1}$.

pendence and correlation length, despite intense computational efforts. Nevertheless, our data clearly show the reported exponential to power-law crossover in the decay of the amplitude of $C_{J}(r, t)$.
The highest temperature at which this decay exhibits a power law tail, is consistent with the temperature $T_{x}$ where $\left\langle t_{w}\right\rangle$ and $\left\langle t_{p}\right\rangle$ first decouple, and the SE relation breaks down (see Sec 3.1). This suggests that the breakdown of the $\mathrm{SE}$ relation is related to a crossover in the features of the facilitation process. We investigate this crossover focussing on the number of jumps triggered by a given jump. This is given by $N_{\operatorname{tr}}(T) \propto \int_{0}^{\infty} n(t, T) d t$, where $n(t, T)=\int C(\mathbf{r}, t) \mathbf{r} d \mathbf{r} \propto A(t, T) \xi^{2}(t, T) d t$, is the number of jumps it triggers at time $t$. As at high temperature the variation of the correlation length is small with respect to that of the amplitude, one can assume $\xi(t, T) \simeq \xi(0, T)$ and estimate $N_{\mathrm{tr}}(T) \propto A(0, T) \xi^{2}(0, T) \tau_{A}(T)$. At low temperature, the integral is dominated by the long time power law behavior of the amplitude and of the correlation length, and the number of triggered events diverges as $N_{\mathrm{tr}}(T, t) \propto \int_{0}^{t} A(t) \xi^{2}(t) d t \propto t^{-a+2 b+1} \propto$ $t^{0.8}$.

\section{Discussion}

We conclude by noticing that the above scenario suggests to interpret facilitation as an infection spreading process, in which a particle is infected each time it jumps. Since each particle can be infected more than once, the relevant infection model is of susceptible-infected-susceptible (SIS) type. In this framework, the exponential to power-law crossover in the decay of the amplitude of $C_{J}(r, t)$ signals a transition from a high temperature resilient regime, in which a single infected site only triggers a finite number of infections, to a low temperature regime in which the number of triggered infection diverges. A complementary interpretation can be inspired by the diffusing defect paradigm $\underline{42} ; \underline{1}$. We suggest that the correlation length of contemporary jumps, $\xi_{J}(0)$, is akin to the typical defect size, which, according to our results, is temperature independent. In the high temperature regime, this is the only relevant correlation length, as defects are rapidly created and destroyed by noisy random fluctuations, before they 
can sensibly diffuse. At low temperature, the effect of noise becomes smaller: the short time correlation length is still dominated by the defect size, $\xi_{J}\left(t<\tau_{A}\right) \simeq \xi_{J}(0)$, whereas its long time behaviour, $\xi_{J}\left(t>>\tau_{A}\right)$, is controlled by the typical distance defects have moved up to time $t$. Further studies are necessary to investigate which of the two interpretations is more appropriate.

\section{Acknowledgement}

We acknowledge financial support from MIUR-FIRB RBFR081IUK, from the SPIN SEED 2014 project Charge separation and charge transport in hybrid solar cells, and from the CNR-NTU joint laboratory Amorphous materials for energy harvesting applications.

\section{References}

[1] L. Berthier and G. Biroli, Rev. Mod. Phys., 2011, 83, 587.

[2] G. Biroli and J.P. Garrahan, J. Chem. Phys. 2013, 138, $12 \mathrm{~A} 301$.

[3] T.R. Kirkpatrick and D. Thirumalai, Rev. Mod. Phys., 2015, 87, 183.

[4] G. A. Appignanesi, J. A. R. Fris, R. A. Montani, and W. Kob, Phys. Rev. Lett., 2006, 96, 05780; B. Vogel, A. Doliwa, A. Heuer, and S. C. Glotzer, J. Chem. Phys., 2004, 120, 4404; R. A. L. Vallee, M. van der Auweraer, W. Paul, and K. Binder, Phys. Rev. Lett., 2006, 97, 217801; J. A. R. Fris, G. A. Appignanesi, and E. R. Weeks, Phys. Rev. Lett., 2011, 10, 065704; C. De Michele and D. Leporini, Phys. Rev. E, 2001,63, 036701.

[5] L. Berthier, G. Biroli, J.-P. Bouchaud, L. Cipeletti, and W. van Saarloos, Dynamical heterogeneities in glasses, colloids, and granular media, Oxford University Press, New York, 2011.

[6] J.P. Garrahan and D. Chandler, Phys. Rev. Lett., $2002, \mathbf{8 9}, 035704$.

[7] J.P. Garrahan and D. Chandler, Proc. Natl. Acad. Sci. U.S.A., 2003, 100, 9710.
[8] D. Chandler and J.P. Garrahan, Annu. Rev. Phys. Chem., 2010, 61, 191.

[9] F. Ritort and P Sollich, Adv. Phys, 2003, 52, 219.

[10] B. Doliwa and A. Heuer, Phys. Rev. E 2003, 67, 030501; A. Heuer, B. Doliwa, and A. Saksaengwijit, Phys. Rev. E, 2005, 72, 021503; C. Rehwald and A. Heuer, Phys. Rev. E 86, 051504.

[11] K. Vollmayr-Lee, J. Chem. Phys., 2004, 121, 4781; K. Vollmayr-Lee and A. Zippellius, Phys. Rev. E, 2006, 72, 041507.

[12] J.W. Ahn, B. Falahee, C. Del Piccolo, M. Vogel and D. Bingemann, J. Chem. Phys., 2013, 138, 12 A527.

[13] H. Shiba,T. Kawasaki, and A. Onuki, Phys. Rev. E, 2012, 86, 041504. T. Kawasaki and A. Onuki, J. Chem. Phys., 2013, 138, 12A514. T. Kawasaki and A. Onuki, Phys. Rev. E, 2013, 87, 012312.

[14] A. Widmer-Cooper, H. Perry, P. Harrowell and D. R. Reichman, Nature Physics, 2008, 4, 711.

[15] E. Lerner, I. Procaccia and J. Zylberg, Phys. Rev. Lett., 2009 102, 125701.

[16] P. Yunker, Z. Zhang, K.B. Aptowicz, A.G. Yodh, Phys Rev. Lett., 2009, 103, 115701.

[17] J. Helfferich, F. Ziebert, S. Frey, H. Meyer, J. Farago, A. Blumen, and J. Baschnagel, Phys. Rev. E., 2014, 89, 042603,042604 .

[18] S. Carmi, S. Havlin, C. Song, K. Wang and H. A. Makse, J. Phys. A: Math. Theor., 2009, 42, 105101.

[19] D. A. Stariolo, J. J. Arenzon and G. Fabricius, Physica A, 2004, 340, 316.

[20] M. Mosayebi, P. Ilg, A. Widmer-Cooper, and E. Del Gado, Phys. Rev. Lett., 2014, 112, 105503.

[21] R. Candelier, A. Widmer-Cooper, J. K. Kummerfeld, O. Dauchot, G. Biroli, P. Harrowell, and D. R. Reichman, Phys. Rev. Lett., 2010, 105, 135702.

[22] A.S. Keys, L.O. Hedges, J.P. Garrahan, S.C. Glotzer, and D. Chandler, Phys. Rev. X, 2011, 1, 021013.

[23] R. Candelier, O. Dauchot and G. Biroli, Phys. Rev. Lett., 2009, 102, 088001. 
[24] S. Gokhale, K. H. Nagamanasa, R. Ganapathy and A.K. Sood, Nature Comm., 2015, 5, 4685.

[25] M. Kitsak, K. L. Gallos, S. Havlin, F. Liljeros, L. Muchnik, H. E. Stanley and H. A. Makse, Nature Physics, 2010, 6, 888.

[26] S. Plimpton, J. Comp. Phys., 1995, 117, 1.

[27] C. N. Likos, Phys. Rep., 2001, 348, 267.

[28] D. J. Durian, Phys. Rev. Lett., 1995, 75, 4780; Phys.Rev. E, 1997, 55, 1739.

[29] D. Paloli, P. S. Mohanty, J. J. Crassous, E. Zaccarelli and P. Schurtenberger, Soft Matter, 2013, 9, 3000.

[30] B. L. Berthier and T. A. Witten, Europhys. Lett., 2009, 86, 10001.

[31] M. L. Manning and J. A. Liu, Phys. Rev. Lett., 2011, 107, 108302.

[32] R. Pastore, A. Coniglio and M. Pica Ciamarra, Soft Matter, 2014, 10, 5724 .

[33] L. Larini, A. Ottochian, C. De Michele and D. Leporini, Nature Physics, 2007, 4, 42.

[34] R. Pastore, A. Coniglio and M. Pica Ciamarra, Sci. Rep., 2015, 5, 11770.

[35] R. Pastore, G. Pesce, A. Sasso and M. Pica Ciamarra, Soft Matter, 2015, 11, 622.

[36] E. W. Montroll and G. H. Weoss, J. Math. Phys., $1965,6,167$.

[37] L. Berthier, D. Chandler, and J. P.Garrahan, Europhys. Lett., 2005, 69, 320; Y. Jung, J. P. Garrahan and D. Chandler, Phys. Rev. E, 2004, 69, 061205.

[38] A. Jaiswal, T. Egami and Y. Zhang, Phys. Rev. B, 2015, 91, 134204.

[39] T. Rizzo Europhys. Lett, 2014, 106, 56003; T. Rizzo and T. Voigtmann, 2014, arXiv:1403.2764. T. Rizzo and T. Voigtmann, 2015, arXiv:1504.06263.

[40] H. R. Schober, C. Oligschleger, and B. B. Laird, J. Non- Cryst. Solids, 1993, 965, 156-158; C. Oligschleger and H. R. Schober, Phys. Rev. B, 1999, 59, 811; W. Kob, et al., Phys. Rev. Lett., 1997, 79,
2827; C. Donati, et al., Phys. Rev. Lett., 1998, 80, 2338; C. Donati et al., Phys. Rev. E, 1999, 60, 3107; S. C. Glotzer, J. Non-Cryst. Solids, 2000, 274, 342.

[41] Y. Zhoua and S.T. Milner, Soft Matter, 2015, 11, 2500 .

[42] J. T. Bendler and M. F. Shlesinger, J. Stat. Phys., 1998, 53, 531. 